\title{
SOPRA ALCUNE QUESTIONI DI GEOMETRIA
}

\author{
DIFFERENZIALE.
}

Nota di C. Burall-Fortl, in Torino.

$$
\text { Aduranza del } 23 \text { genualo } 1898 .
$$

Nelle mie due Note a Il metodo del Grassmann nella geometria proiettiva (*), ho cercato dimostrare come dagli elementi del Calcolo Geometrico di Grassmann e dagli elementi della teoria delle corrispondenze lineari, si possano dedurre, e in modo assai semplice, le ordinarie propriets che si studiano nella geometria proiettiva. In un mio libro (**), di recente pubblicazione, ho pure cercato dimostrare come le ordinarie proprietà delle curve, degli inviluppi e delle superficie rigate, si ottengano in modo semplice ed elegante dagli elementi del calcolo di Grassmann. In questa breve Nota mi propongo identico scopo per ció che riguarda la teoria delle superficie, facendo vedere come alcune ben note proprietà delle superficie possano ottenersi per via semplice ed elementare, senza presupporre note questioni speciali di analisi infinitesimale e senza far uso,

(") Questi Rendiconti, t. X (1896) e t. XI (1897).

(") Introduction d la Giometrie differentielle suivant la methode de $H$. Grassmann. (Paris, Gauthier-Villars, 1897 ). Citero questo libro con l'indicszione m.l. 
- direttamente, o indirettamente, di sviluppi complicati (*). In si piccola mole non posso aver certamente riunite tutte le eleganti proprietì di cui é ricea la geometria differenziale : ne ho scelee alcune, ma si puo facilmente comprendere come con i medesimi mezzi-che sono di una estrema semplicita - si possano trattare le altre. Avró raggiunto lo scopo che mi sono proposto, se ill lettore potra convincersi che multe delle questioni dell'attuale Geometria differenziale superiore, possono entrare a far parte delle ordinarie ed elementari applicazioni geometriche del Calculo.

1. Sia $P(u, v)$ un punto funzione continua, insieme alle sue derivate, delle variabili $u, v$ in un dato campo, nel quale supporremo sempre che sia

$$
P^{\prime} P^{\prime} \neq 0 \text { : }
$$

il punto $P$ descrive allora, col variare di $u, v$ nel dato campo, una superficie in ogni cui punto $P$ il piano tangente è parallelo al bivettore $P_{.}^{\prime} P_{\text {. }}^{\prime}$.

Se poniamo

$$
K=\mid \frac{P_{u}^{\prime} P^{\prime}}{\bmod \left(P_{*}^{\prime} P_{*}^{\prime}\right)}
$$

allora $K$ \& un vettore unità parallelo alla normale alla superficie in $P$ e il cui verso puó esser preso come verso positivo sulla normale. Il vettore $d P$ è normale a $K$, perche $d P$ giace sul piano tangente in $P$, e quindi$$
d P \mid K=0
$$

(") Il lettore, per rendersi esatto conto deiia semplicita delle dimostrazioni, deve osservarte che io mi valgo (insieme ag'i eiamenti della teoria delle corrispondenze lineari) solo della parte teorica contenuta sel mio libro gid eitato, parte che, pur contenendo implicitaneate tutli i sistemi di coordinate lineari, puo essere comodamente svo'ta in to lezioni, e non richiede altre nozioni all'infuori del'a geometria elementare, dell'a'goritmo algebrico, e degli elementi di Calcolo differenzia!e e integrale. 
SOPRA ALCUNE QUESTIONI DI GEONETRIA DIFFERENZIALE.

che differenziata da

(2)

$$
d^{2} P|R=-d P| d K
$$

Arendosi ancora in particolare che

$$
P^{\prime} \mid K=0 \quad \text { e } \quad P_{0} \mid K=0
$$

si ha, derivando la prima rispetto a $v$ e la seconda rispetto ad $u$,

$$
P_{4}^{\prime}\left|K_{*}^{\prime}=P_{*}^{\prime}\right| K_{*}^{\prime}
$$

2. Per ogni relazione $f(u, v)=0$ tra $u$ e $v, P$ descrive una linea sulla superficie: questa linea elinea di curvatura, quando la rigata descritta da $P K$ è sviluppabile, cioè quando (m. 1, pag. 99) $d(P K) \cdot d(P K)=0$. Osservando che $d(P K)=(d P) K+P(d K)$ si ha che la retta $P K$ descrive una sviluppabile quando $K(d P)(d K)=0$, ciot quando i vettori $K, d P, d K$ sono complanari : ma essendo $K$ un vettore unita, $d K$ e normale a $K$ e giace quindi sul piano tangente in $P$, e per cio la precedente condizione equivale al parallellismo dei vettori $d P, d K$, cioè a

$$
(d P)(d K)=0
$$

che è quindi l'equazione differenziale delle linee di curvatura.

Abbiamo gid osservato che il vetrore $d K$ sta (qualunque sieno gli incrementi $d u, d v$ ) sul piano tangente in $P$, e che quindi $i$ vettori $d P, d K$ sono complanari. Possiamo allora considerare, sul piano tangente in $P$, l'omografia

$$
\sigma=\left(\begin{array}{ll}
K_{*}^{\prime}, & K_{v}^{\prime} \\
P_{w}^{\prime}, & P_{v}^{\prime}
\end{array}\right)
$$

Dall'essere

$$
d P=P_{v}^{\prime} d u+P_{v}^{\prime} d v \text { e } d K=K_{u}^{\prime} d u+K_{v}^{\prime} d v
$$

Rend. Circ. Matem., t. XII, parte 1*.-Stampato il 19 marzo 1898 . 
si deduce che

$$
\sigma(d P)=d K \text {. }
$$

Se $i$ e l'operazione che nel piano tangente in $P$ fa ruotare ogni vettore di un angolo retto ( $e$ il verso positivo della rotazione pud esser determinato dipendentemente dal verso di $K$ ), allora $i \sigma$ t una omografia per il cui invariante (") si ha:

$$
\text { inv. } i \sigma=\frac{P_{0}^{\prime}\left(i K_{0}^{\prime}\right)-P_{0}^{\prime}\left(i K_{\bullet}^{\prime}\right)}{P_{*}^{\prime} P_{*}^{\prime}},
$$

che per la (3) da

$$
\text { inv. } i \sigma=0 \text {. }
$$

L'omografia io ed dunque una involuzione: ma questa ammette - una sola o infinite coppie di raggi coniugati ortogonali, e quindi $\sigma$, o ammette due soli raggi uniti ortogonali, o tutti i suoi raggi sono uniri. $\mathrm{D}_{2}$ questo e dalla $(4)$ e (7) risulta che : per ogni punto $P$ della superficie o passano due sole linee di curvatura che ivi si tagliano ad angolo retto, o ve ne passano infirite (**).

() Cfr. G. Peano: . Trasformazioni lineari dei veltori di un pianos. Atti Acc. Torino, 1895 .

(⿻) Osservando: che

$$
\begin{gathered}
d P \mid d P=\left(P_{u}^{\prime}\right)^{2} d u^{2}+2\left(P_{u}^{\prime} \mid P_{v}^{\prime}\right) d u d v+\left(P_{v}^{\prime}\right)^{2} d v^{2} \\
-d^{2} P|K=d P| d K=\left(P_{a \mid}^{\prime} K_{0}^{\prime}\right) d u^{2}+2\left(P^{\prime} \mid K_{v}^{\prime}\right) d u d v+\left(P_{v}^{\prime} \mid K_{v}^{\prime}\right) d v^{2}:
\end{gathered}
$$

che il discriminante della forma differenziale quadratica \& $P \mid d P$ \&

$$
\left(\bmod P_{n}^{\prime}\right)^{2}\left(\bmod P_{v}^{\prime}\right)^{2}-\left[\bmod P_{*}^{\prime} \cdot \bmod P_{\sigma}^{\prime} \cos \left(P_{*}^{\prime}, P_{\vartheta}\right)\right]^{2}=\left[\bmod \left(P_{*}^{\prime} P_{*}^{\prime}\right)\right]^{2}:
$$

che, se le linee corrispondenti a valori costanti di $u$ o di $v$ sono linee di curvatura ortogonali, sono nulli $\mathrm{i}$ coefficienti di $d u d v$ nelle due forme $d P ! d P, d P \mid d K$ : allora il lettore pub confrontare la dimostrazione geometrica da noi data del noto tcorema sull'esistenza delle linee di curvatura, con l'ordinaria dimostrazione basata su! noto teorema di analisi: se di due forme differenziali quadratiche una almeno é definita allora si puo, $O$ in un sol modo o in infinti modi, cambiare lẹ variabili in guisa che si annullino $i$ coefficienti medii delle due forme. 
SOPRA ALCUNE QUESTIONI DI GEOMETRIA DIFFERENEIALR. IIS

3. Se $s$ é l'arco della curva descritta da $P(d s=\bmod d P$ ), allora per le formule di Frenet (m. l. pag. 121) si ha che $d^{3} P / d s^{2}$ vale il prodotto della prima curvatura in $P$ per un vettore unitd $N$ parallelo alla normale principale e di verso determinato (eccettuati certi punti singolari che noi non consideriamo). Essendo p l'angolo dei vettori $N, K$, dizmo alla prima curvatura $r / p$ (curvatura assoluta), un segno determinato ponendo

$$
-\frac{d^{2} P}{d s^{2}} \mid K=\frac{1}{\rho} \cos \varphi,
$$

ovvero per la (2)

$$
\frac{d P}{d s} \mid \frac{d K}{d s}=\frac{\mathrm{I}}{\rho} \cos \varphi \text {. }
$$

Il valore assoluto della curvitura della sezione normale in $P$, corrispondente alla direzione $d P$, vaie il rapporto tra il modulo della componente normale di $d^{2} P$ [la qual compnnente è $\left(d^{2} P \mid K\right) K$, ovvero per la (2), $-(d P \mid d K) K]$ e il modulo di $d P$ : attribuiamo un segno 2 questa curvatura, curvatura normale, $r / r$, ponendo

$$
\frac{I}{r}=\frac{d P \mid d K}{d P \mid d P}=\frac{d P}{d s} \mid \frac{d K}{d s} \text {. }
$$

Questa combinata con 13 (8) dd

$$
p=r \cos \varphi
$$

che dimostra il teorema di Meunier.

Per la curvatura tangenziale (o geodetica) $\mathrm{I} / \mathrm{g}$ abbiamo

$$
\frac{1}{g}=\frac{\operatorname{sen} \varphi}{p}=\frac{\operatorname{tang} \varphi}{r}
$$

dalla quale si deduce subito che: il raggio di curvasura tangenziale 
di un parallelo di una superficie di rivoluzione i uguale alla parte di tangente al meridiano compresa fra il punto di contatio e l'asse.

La superficie che si considera sia la sviluppabile osculatrice di una curva gobba $Q$ per la quale i vettori unitd $T, N, B$ sono quelli che compariscono nelle formule di Frenet. Siz $Q_{\mathrm{r}}$ il punto che descrive una curva piana di egual arco e di egual curvatura (Hessione) di Q. Posto

$$
P=Q+b T \quad \text { e } \quad P_{\mathrm{s}}=Q_{\mathrm{t}}+b T_{\mathrm{s}}
$$

il punto $P_{3}$ descrive la trasformata di $P$ quando si distende la sviluppabile nel piano (m. l. pag. $1_{1}$ ), e le curve $P, P_{1}$ hanno egual arco. Per le formule di Frenet si ha

$$
\frac{d^{2} P}{d s^{2}}=x T+y N+z B \text { e } \frac{d^{2} P}{d s^{2}}=x T_{3}+y N_{1},
$$

e non abbiamo bisogno di calcolare effettivamente $i$ numeri $x, y, z$. Da queste formule si ha per le curvature in $P$ \& $P_{\mathfrak{r}}$,

$$
\frac{1}{p}=\sqrt{x^{2}+y^{2}+z^{2}}, \quad \frac{1}{p_{1}}=\sqrt{x^{2}+y^{2}}:
$$

ma poicht

$$
\frac{d^{3} P}{d s^{2}} B=x T B+y N B
$$

e il vettore $B$ \& parallelo a $K$, si ha, prendendo i moduli dei due membri,

$$
\frac{\operatorname{sen} \varphi}{P}=\frac{1}{P_{3}}
$$

la quale dimostra un noto teorema di Ca a $_{\text {l la }}$ (Comptes Rendus, 1843) che si suole anche enunciare dicendo: la curvatura tangenziale in $P$ vale la curvatura ordinaria della trasformata piana. Se la sup. considerata d un cono descritto dalla retta $Q T$, allora la precedente 
SOPRA ALCUNB QUESTIONI DI GEOMETRIA DIFFERENZIALE. $\$ 17$ dimostrazione sussiste essendo $Q$ un punto fisso e sostituendo all' elemento di arco di $Q$ il mod $d T$; per un cilindro si supponga essere la curva $Q$ una sezione retta della sup., $Q T$ la binormale, $Q N$ la tangente e $Q B$ la normale principale in $Q$ (m.l. pag. 126) (").

Altra forma notevole della curvatura tangenziale in $P$, per una superficie qualunque, e

$$
\frac{1}{g}=\frac{d P}{d s} \frac{d^{2} P}{d s^{2}} K \quad \text { ovvero } \quad \frac{1}{g}=\frac{1}{P} T N K
$$

(") Ecco un altra dimostrazione del teorema di $\mathrm{C}_{2} \mathrm{ta}_{2}$ a n. La sup. sia descritta dalla retta $P I$, ove $I$ è un vettore unita funzione dell'arco s di $P$. Distesa la sup. su di un piano sieno $P_{1}, I_{1}$ gli elementi corrispondenti a $P, I$. Si ha

$$
\pi I=T_{1} \mid I_{3},
$$

essendo i vettori $T, N, B$ relativi alla curva $P$, e quindi, derivando,

$$
\frac{1}{p} N\left|I+T \frac{d I}{d s}=\frac{1}{\rho_{1}} N_{1}\right| I_{t}+T_{\mathrm{s}} \mid \frac{d I_{\mathrm{t}}}{d s} .
$$

Facciamo lo sviluppo sul piano tangente lungo $P I$, facendo coincidere $P_{3}$ e $I_{1}$ con $P$ ed $I$ : risulta che per il valore considerato di $s, T_{1}$ ed $I_{z} / d s$ coincidono con $T$ e $d I / d$ s. Osservando che se $U, V, W$ sono vettori si ha

$$
U V W=(U 1 W) V-(U) V \mid W,
$$

essendo i prodotti regressivi sviluppati sul piano all'infinito, allora, moltiplicando 12 formula precedente per $\mid T$, si ottiene, dogo aver soppressi i termini nulli e quelli comuni ai due membri,

$$
\frac{1}{P^{\prime}} N_{l} T I=\frac{1}{\rho_{1}} N_{1} \mid T I_{L^{\circ}}
$$

Se $\theta$ e l'angolo, non nullo, che $T$ fa con $I$, la formula ora ottenuta da

$$
\frac{1}{\rho} \operatorname{sen} \varphi \operatorname{sen} \theta=\frac{1}{\rho_{z}} \operatorname{sen} \theta \text {, }
$$

(perche $\mid T I$ e parallelo a $K$ ) ovyero

$$
\frac{\operatorname{sen} \varphi}{p}=\frac{1}{p r}
$$


dalla quale si deducono facilmente note proprietà delle linee 2 curvatura tangenziale costante (circoli geodetici secondo il Darboux).

Se le linee coordinate $u, v$ sono linee di curvatura ortogonali, allora per le curvature normali $1 / r_{1}, 1 / r_{2}$ corrispondenti alle direzioni principali $P_{n}^{\prime}, P_{*}^{\prime}$ si ha dalla (9)

$$
\frac{1}{r_{1}}=\frac{P_{d}^{\prime} \mid K_{u}^{\prime}}{P_{0}^{\prime} \mid P_{0}^{\prime}}, \quad \frac{1}{r_{1}}=\frac{P_{0}^{\prime} \mid K_{\dot{v}}^{\prime}}{P_{v}^{\prime} \mid P_{0}^{\prime}}
$$

le quali, per essere i vettori $K_{a}^{\prime}, K_{v}^{\prime}$ rispettivamente paralleli ai vettori $P_{*}^{\prime}, P_{.}^{\prime}$ [formula (4)] dinno

$$
K_{*}^{\prime}=\frac{1}{r_{1}} P_{*}^{\prime}, \quad K_{0}^{\prime}=\frac{1}{r_{2}} P_{*}^{\prime}
$$

Se $P$ z un ombelico $\left(r_{1}=r_{2}\right)$, allora, in virtù delle $(x x)^{\prime}$, l'omografia o si riduce al numero $s ! r_{1}, e$, in conseguenza, per il punto $P$ passano infinite linee di curvatura. Si ha pure facilmente che : le superficie per le quali ogni linea í lines di curvatura sono solamente quelle per le quali ozni punto $P$ i un ombelico. Per ogni punto di tali superficie deve esistere un numero $r / r$, funzione di $u, v$, tale che

$$
d R=\frac{\mathrm{I}}{r} d P:
$$

questa, differenziata e moltiplicata per $d P$ (che non e nullo), dd

$$
\left(d^{2} R-\frac{1}{r} d^{2} P\right) d P=0:
$$

non essendo, in generale, i vettori $d P, d^{2} P, d^{2} R$ complanari, la precedente relazione $\&$ sodisfatta solamente quando per ogni valore di $u, v$ si ha che $d^{2} R-\frac{1}{r} d^{2} P=0$; ora questa combinata con la penultima formula differenziala, dt $\left(d \frac{1}{r}\right) d P=0$, orvero 
SOPRA ALCUNE QUESTIONI DI GEOMBTRIA DIFFERENZIALB.

$d\left(\frac{I}{r}\right)=0$, o $1 / r=$ cost. Se $\mathrm{r} / r=0$, allora $d K=0$ e $K$ d un vettore costante, cioes la sup. descritta da $P$ è un piano: se $r / r \neq 0$, allora l'equazione $d K=\frac{I}{r} d P$, integrata, da $P=O+r K$, ove $O$ è un punto fisso, e quindi $P$ descrive una sfera. Segue da ció che: soltanto per $i$ piani e per le sfere ogni linea l' linea di curvatura (*). Dalle formule (9), (II)' si ha che

$$
\frac{I}{r}=\frac{I}{r_{1}}\left(P_{v}^{\prime}\right)^{2}\left(\frac{d u}{d s}\right)^{2}+\frac{1}{r_{2}}\left(P_{*}^{\prime}\right)^{2}\left(\frac{d v}{d s}\right)^{2}:
$$

detto $\psi$ l'angolo che $d P$ fa con $P_{*}^{\prime}$, allora, dallo sviluppo del prodotto interno $\frac{d P}{d s} \mid P_{\text {. }}^{\prime}[\mathrm{cfr}$. formule (6)], si ha subito che $\mathrm{i}$ coefficienti di $I / r_{1}, I / r_{2}$ valgono, rispettivanente, $\cos ^{2} \psi, \operatorname{sen}^{2} \psi$ e quindi la formula precedente ds

$$
\frac{I}{r}=\frac{\cos ^{2} \psi}{r_{1}}+\frac{\operatorname{sen}^{2} \psi}{r_{2}}
$$

che la formula di Eulero, dalla quale deduconsi le ordinarie proprietd della Indicatrice di $D$ upin.

Quando le curvature principali in $P$ non sono nulle, allora l'involuzione delle direzioni dei diametri coniugati dell'indicatrice di $D u p i n$ coincide con l'involuzione io come risulta subito dalle formule (II)'. Da cio si deduce che: in ogni punto $P$ di una curva della sup., la tangente e la generatrice della sviluppabile circoscritta lungo essa curva sono diametri coniugati della indicatrice di $D u p i n$.

(") Quando in ogni punto $P_{1} r_{1}=r_{2}$, allora $i$ coefficienti delle forme differenziali quadratiche $d P|d P, d P| d K$, sono proporzionali, e viceversa. L'indeterminazjone considerata nel teorema di aralisi citato nella nota $a 1 n^{\circ} 2$, ha pure luogo solamente nel caso de'la proporzionalita dei coefficienti delle due forme.

Si osservi che l'essere $d P \mid d K$ identicamente nulio, caratterizza il fatto che il punto $P$ descrive un piano, il che pub anche esprimersi dicindo $\left(n^{\circ} 4\right)$ : solamente ual piano ognt linea d asintotica. 
Infatti : il piano tangente in $P$ z la posizione della forma

$$
P \mid K
$$

la generatrice della sviluppabile in questo piano sta anche sul piano (m. 1. pag. 85)

$$
d(P \mid K)=d P|K+P| d K=P \mid d K,
$$

e quindi la generatrice considerata passa per $P$ ed d normale a $d K$, ciod ha la direzione coniugata di $d P$ rispetto all'involuzione $i \sigma(*)$.

4. Se le linee coordinate $u$, v sono linee di curvatura ortogonali, allora dalle (5), (II)' si ha

$$
\sigma=\left(\begin{array}{cc}
\frac{I}{r_{1}} P_{n}^{\prime}, & \frac{I}{r_{1}} P_{0}^{\prime} \\
P_{n}^{\prime}, & P_{0}^{\prime}
\end{array}\right):
$$

quindi per il determinante (rapporto delle aree corrispondenti) e per l'invariante di $\sigma$ si ha

$$
\text { det. } \sigma=\frac{1}{r_{1} r_{2}}, \quad \text { inv. } \sigma=\frac{1}{r_{1}}+\frac{1}{r_{2}},
$$

le quali dicono che il determinante e l'invariante di o sono, rispettivamente, la curvatura di Gauss (o curvatura totale) e la curvatura media della sup. nel punto $P$.

Se la sup. descritta da $P$ e a curvatura nulla, allora, a causa della continuità supposta per $P$ e per le sue derivate, 0 I $/ r_{1}$ o I $/ r_{2}$ d costantemente nullo, ciod la sup. el rigata (m. l., pag. 106): ma se $P$ descrive una generatrice di una rigata gobba la retta $P K$ non

(") Risulta pure facilmente che $\mathrm{i}$ sistemt coniugati sono quelli per $\mathrm{i}$ quali $P^{\prime}\left|R_{:}^{\prime}=P_{!}^{\prime}\right| K_{:}^{\prime}=0$, ovvero $\frac{d^{2} P}{d u d v \mid} \mid K=0$. 
descrive una sviluppabile, e quindi: le sole superficie a curvatura nulla sono le rigate sviluppabili.

Sia $O$ un punto fisso; allora il punto $Q=O+K$ descrive la rappresentazione sferica della sup. $P$. Se $d P, d ' P$ sono due direzioni uscenti da $P$, le direzioni corrispondenti nella rappresentazione sferica sono $d K, d^{\prime} K$. Ora, affiache da

$$
d P \mid d^{\prime} P=\left(P_{u}^{\prime}\right)^{2} d u d^{\prime} u+\left(P_{v}^{\prime}\right)^{2} d v d^{\prime} v=0
$$

si abbia come conseguenza

$$
d K \mid d^{\prime} K=\frac{I}{r_{1}^{2}}\left(P_{u}^{\prime}\right)^{2} d u d^{\prime} u+\frac{1}{r_{2}^{2}}\left(P_{v}^{\prime}\right)^{2} d v d^{\prime} v=0,
$$

es necessario e sufficiente the sia

$$
\frac{\mathrm{I}}{r_{1}^{2}}-\frac{\mathrm{I}}{r_{2}^{2}}=0 \text {, orvero }\left(\frac{\mathrm{I}}{r_{2}}+\frac{\mathrm{I}}{r_{2}}\right)\left(\frac{\mathrm{I}}{r_{1}}-\frac{\mathrm{I}}{r_{2}}\right)=0 \text {; }
$$

per ciò : tutte le direzioni ortogonali in $P$ si conservano ortogonali nella rappresentazione sferia, solamente, per gli ombelichi della sup. e per tutti $i$ punti delle superficie a curvatura media nulla (*).

Se il punto $P$ descrive una asinotica, cioes una curva il cui piano osculatore in $P$ coincide col piano tangente in $P$, allora it bivettore $d P . d^{2} P$, che ed parallelo al piano osculatore in $P$, d parallelo al bivettore $\mid K$ : ma poiché $d P$ e parallelo a $\mid K$, si ha che l'equazione differenziale delle asintolicbe eे

$$
d^{3} P \mid K=0
$$

ovvero per la (2)

$$
d P \mid d K=0
$$

(") In questi due casi o $\sigma$ si riduce ad un numero, $0 \sigma$ un'involuzione. Cio dimostra il teorema di geometria prolettiva: se due fasei di raggi proiettivi e sovrapposti hanno due raggi uniti ortogonali, al'ora, dire che a due raggi ortogona'i dell'un fascio corrispondono raggi ortogonali nell'altro fascio, equivale a dire che $\mathrm{i}$ duc fasei formaro una involuzione.

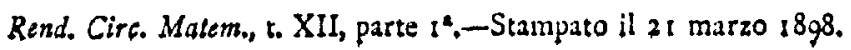


la quale confrontata con $\mathrm{la}$ (9) dice che: le asintoticlse sono le linee a curvatura normale nulla (*).

Il vettore $K$ e parallelo alla binormale dell'asiototica in $P$, e quindi, per le formule di Frenet, $\left(\frac{d K}{d s}\right)^{2}$ e il quadrato della torsione in P. Ora si ha [cfr., quanto abbiamo detto per ottenere la (12)]

$$
\left(\frac{d K}{d s}\right)^{2}=\left(K_{v}^{\prime}\right)^{2}\left(\frac{d u}{d s}\right)^{2}+\left(K_{v}^{\prime}\right)^{2}\left(\frac{d v}{d s}\right)^{2}=\frac{\cos ^{2} \psi}{r_{3}^{2}}+\frac{\operatorname{sen}^{2} \psi}{r_{1}^{2}},
$$

la quale confrontata con la (12) ove si ponga $1 / r=0 \mathrm{da}$

$$
\left(\frac{d K}{d s}\right)^{2}=-\frac{1}{r_{3} r_{2}}
$$

che dimostra il teorema di Enneper. Se osserviamo che

$$
d P \mid d K=\left(P_{*}^{\prime} K_{*}^{\prime}\right) d u^{2}+\left(P_{*}^{\prime} \mid K_{*}^{\prime}\right) d v^{2}
$$

(essendo $u, v$ coordinate coniugate) e che per $d P \mid d K=0$ si hanno per $d u / d v$ due valori di egual modulo e di segno contrario, risulta subito che : se per $P$ passano due asintotiche, le loro torsioni in $P$ sono eguali e di segno contrario.

5. Da una nota forma (**) della variazione dell' $\int d s$

$$
\delta \int_{Q}^{R} d s=\left.\right|_{Q} ^{R} \frac{d P}{d s}\left|\delta P-\int_{Q}^{R} \frac{d^{2} P}{d s^{2}}\right| \delta P d s
$$

( $)$ Se per $P$ passano due assintotiche queste hanno per tangenti i raggi doppi dell'involuzione $i \sigma$. Risulta che per ogni punto di una sup. 2 curvatura media nulla passano due asintotiche che ivi si tagliano ad angolo retto (ćr. nota precedente).

Si puo anche dire che il punto $P$ deserive una asintotica, solo quando la linea descritta da $P$ \& di stringimento per la rigata $P K$. Infatti il punto centrale sulia retta $P K$ \&, posto $U=i K(d K)$, (m. l., pag. 97),

$$
P K U .(P d K-K d P)=P K U(d K) . P-P K U(d P) . K \text {; }
$$

e perchè sia $K U(d P)=0$ es necessaric e sufficiente che sia $d P ! d K=0$ poichè $U$ \& appunto un vettore, del piano tangente, normale $a d K$.

(*) P e a o : Lefioni di Analisi Infinitesimale, vol. Il, pag. 321. 
SOPRA ALCUNE QUESTIONI DI GEOMETRIA DIFFRRENZIALE.

risulta subito che in ogni punto di una geodetica, la normale principale coincide con la normale alla superficie. Se $P$ descrive una geodetica, e l'arco $Q R$ ha lunghezza costante, allora

$$
\int_{Q}^{R} \frac{d P}{d s} \mid \delta P=0
$$

e quindi, se $Q$ descrive una traiettoria ortogonale delle geodetiche descritte da $P$ col variare di $Q$, anche $R$ descrive una traiettoria ortogonale delle stesse geodetiche, e si ha cost una nota proprietz delle linee geodeticamente parallele e dei circoli geodetici.

Se il punto $P$ descrive una geodetica allora $\frac{d^{2} P}{d s^{2}}$, che es parallelo alla normale principale, es parallelo a $K$ e quindi

$$
K \frac{d^{2} P}{d s^{2}}=0
$$

el l'equazione differenziale delle geodeliche. Sotto altra forma si ha per la stessa equazione

$$
\text { K. } d P \cdot d^{3} P=0
$$

perchè il piano osculatore in $P$ è parallelo al bivettore $d P . d^{2} P$.

Se $P$ descrive una geodetica che sia pure linea di curvatura, cioè per la quale si abbia $d P . d K=0$, allora differenziando la (13) si ha $K . d P . d^{3} P=0$, che combinata con la (13) dice che $i$ vettori $d P, d P^{2}, d^{3} P$ sono complanari, cioe che (m. l., pag. 67) $P$ descrive una curva piana. Dunque : ogni lined geodetica che $i$ linea di curvatura ¿ una curva piana. Se $P$ descrive una geodetica piana, allora i vettori $K, d P, d^{2} P, d^{3} P$ sono complanari, e quindi differenziando la (13) si ha $d K . d P . d^{2} P=0$ : questa e verificata quando $d^{2} P=0$, o quando il vettore $d K$ sta sul piano osculatore della curva, cioes quando $d P . d K=0$. Dunque : ogni linea geodetica piana o é una retta o è una linea di curvatura.

Il punto $P$ descriva una geodetica (non rettilinea) e siz $B$ if 
vettore unitd parallelo alla binormale in $\sigma$ : si ha, determinando anche il verso di $B$,

$$
B=\mid \frac{d P}{d s} K,
$$

da cui si deduce

$$
\frac{d B}{d s}=\int \frac{d^{2} P}{d s^{2}} K+\int \frac{d P}{d s} \frac{d K}{d s}=\mid \frac{d P}{d s} \frac{d K}{d s},
$$

poiche $d^{3} P / d s^{2}$ è parallelo a $K$. Essendo il modulo di $d B / d s$ il valore assoluto della torsione della geodetica in $B$, poniamo, attribuendo cosi un segno a questa torsione $1 / \tau$,

$$
\frac{1}{\tau} K=\frac{d P}{d s} \frac{d K}{d s}
$$

la quale, in virtù della (4) dimostra che: le linee di curvatura sono le linee che banno in ogni loro punto la torsione geodetica nulla.

Se $u, v$ sono linee di curvatura ortogonali si ba dalla ( $\left.r_{4}\right)$

$$
\frac{I}{\tau} K=\left|\left(P_{*}^{\prime} K_{v}^{\prime}+P_{v}^{\prime} K_{*}^{\prime}\right) \frac{d u}{d s} \frac{d v}{d s}=\left(\frac{1}{r_{1}}-\frac{I}{r_{2}}\right) \frac{d u}{d s} \frac{d v}{d s}\right| P_{*}^{\prime} P_{v}^{\prime}=
$$

$=\left(\frac{\mathrm{l}}{r_{1}}-\frac{1}{r_{1}}\right) \frac{d u}{d s} \frac{d v}{d s}\left(\bmod P_{2}^{\prime}\right)\left(\bmod P_{v}^{\prime}\right) K=\left(\frac{1}{r_{1}}-\frac{1}{r_{2}}\right) \operatorname{sen} \psi \cos \psi K$,

da cui si àeduce

$$
\frac{1}{r}=\frac{1}{2}\left(\frac{1}{r_{3}}-\frac{1}{r_{3}}\right) \operatorname{sen} 2 \psi ;
$$

e questa dimostra, insieme ad altre proprietd, che: due geodetiche ortogonali banno nel loro punto d'iniontro torsioni eguali in valore assoluto e di segno contrario.

Il punto

$$
P_{8}=P-r_{1} K
$$


descrive una falda dell'evoluta della sup. $P\left({ }^{*}\right)$. Se $u$, v sono linee di curvatura ortogonali, allora dalle (11)' si ha

$$
\begin{gathered}
\frac{d P_{1}}{d u}=P_{u}^{\prime}-r_{1} K_{u}^{\prime}-\frac{d r_{1}}{d u} K=-\frac{d r_{1}}{d u} K \\
\frac{d P_{1}}{d v}=P_{v}^{\prime}-r_{1} K_{v}^{\prime}-\frac{d r_{1}}{d v} K=\left(r_{1}-r_{1}\right) K_{v}^{\prime}-\frac{d r_{1}}{d v} K
\end{gathered}
$$

quindi

$$
\frac{d P_{1}}{d u} \frac{d P_{2}}{d v}=\left(r_{1}-r_{2}\right) \frac{d r_{1}}{d u} K K_{v}^{\prime}(* *)
$$

Risulta da questa che la normale in $P$, all'evoluta es parallela a $P_{*}^{\prime}$; ma la tangente in $P_{1}$ alla curva considerata es parallela a $K$ e il piano osculatore è parallelo a $K K_{4}^{\prime}$ e in conseguenza : gli spi-

(') Se $u, v$ sono linee di curvatura ortogonali, allora

$$
\begin{aligned}
& P K\left[P_{u}^{\prime} K+P K_{u}^{\prime}\right]=P P_{u}^{\prime} K . K-K P K_{u}^{\prime} \cdot P= \\
& P K K_{u}^{\prime}\left\{P-\frac{P K P_{u}^{\prime}}{P K K_{u}^{\prime}} K\right\}=P K K_{*}^{\prime}\left\{P-r_{t} K\right\},
\end{aligned}
$$

il che prova essere $P_{1}$ il punto che descrive lo spigolo di regresso della sviluppabile descritta da $P K$ quando $P$ descrive la linea di curvatura $v=$ cost. Si osservi che il piano osculatore in $P_{z}$ è

$$
K\left(P K_{u}^{\prime}+P_{*}^{\prime} K\right)=-P K K_{u}^{\prime},
$$

cioè il piano normale alla sup. $P$ uscente da $P$ e parallelo alla direzione $P_{*}^{\prime}$

(") Se osserviamo che

$$
\frac{d^{2} P_{1}}{d u d v}=-\frac{d r_{1}}{d u} K_{v}^{\prime}-\frac{d^{2} r_{1}}{d u d v} K
$$

e quindi che

$$
\frac{d^{2} P_{1}}{d u d v} \mid K_{u}^{\prime}=0
$$

risulta subito che: nelle due falde dell'evoluta le linee corrispondenti alle lioee di curvatura della sup. $P$, formano un sistema coningato. 
goli di regresso delle sviluppabili lungo delle normali ad una superficie lungo le sue linee di curvatura, sono geodetiche della superficie evoluta (").

Il punto $P$ descriva una sup. di rivoluzione di cui l'asse e la posizione della forma (costante) di seconda specie $a$ ad invariante nullo e di modulo uno. Se $P$ descrive sulla sup. una linea per ogni punto della quale la normale principale giace nel piano meridiano corrispondente, allora

$$
P \frac{d^{2} p}{d s^{2}} a=0
$$

da cui si deduce che

$$
P \frac{d P}{d s} a=\text { cost. }
$$

perché la derivata rispetto ad $s$ del primo membro di questa è appunto il primo membro della formula precedente. Decomposto il rettore $\frac{d P}{d s}$ nella somma di due vettori, uno parallelo alla tangente al parallelo che passa per $P$ e l'altro parallelo alla tangente al meridiano che passa per $P$, si ha

$$
P\left(\frac{d P}{d s}\right)_{p} a+P\left(\frac{d P}{d s}\right)_{m} a=\text { cost.; }
$$

ma poiche $P\left(\frac{d P}{d s}\right)_{m} a=0$, si ha ancora

$$
P\left(\frac{d P}{d s}\right)_{p} a=\text { cost. }
$$

Orz il modulo di $P\left(\frac{d P}{d s}\right)$, vale il seno dell'angolo che $d P$ fa

(") Dalle formule precedenti si deducono assai faci.mente a!tre note proprietż della superficie evoluta. 
SOPRA ALCUNE QURSTIONI DI GEOMETRIA DIFFERENZIALE.

col meridiano uscente da $P$, e poiché la minima distanza delle due linee ortogonali $P\left(\frac{d P}{d s}\right)_{p}$, $a$, e il raggio del parallelo che passa per $P$, la formula precedente dimostra il teorema di Clairaut (*), e anzi dimostra ancora che l'essere costante la quantita indicata nel teorema di Clairaut è condizione che caratterizza i paralleli e le geodetiche della superficie di rivoluzione.

6. I metodi fin qui adoperati permettono di risolvere assai facilmente anche le questioni relative alle congruenze di rette. Ne daremo qualche esempio.

(") Questa dimostrazione es una semplice traduzione in linguaggio geometrico di una dimostrazione che il prof. $F$. Castell a no da, da alcuni anni, del teorema di Cla iraut nelle sue lezioni di Meccanica razionale.

Un'altra dimostrazione assai semplice dillo stesso teorema el la seguente. Sia $u$ l'arco di meridiano e $v$ l'angolo che il semi-meridiano fa con un semi-meridiano fisso. La quantití considerata nel teorema di Clairaut è

$$
d P^{-} P_{v}^{\prime}
$$

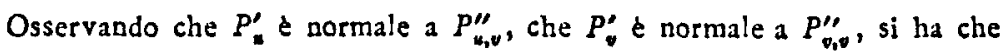

$$
\frac{d}{d s}\left(\frac{d P}{d s} \mid P_{v}^{\prime}\right)=\frac{d^{\prime} P}{d s^{2}} P_{v}^{\prime}+\left(P_{u}^{\prime}\left|P_{v, v}^{\prime \prime}+P_{v}^{\prime}\right| P_{v, v}^{\prime \prime}\right) \frac{d u}{d s} \frac{d v}{d s} ;
$$

se $O$ é il centro del parallelo e $r$ ne è il raggio, allora $P_{v, v}^{\prime \prime}$ il prodotto di $r$ per il vettore unità parallelo a $P-O$ e quindi $P_{v}^{\prime \prime} \mid P_{v, v}^{\prime \prime}=-r \operatorname{sen}(K, O-P)$, ed essendo $P_{u, v}^{\prime \prime}$ il prodotto di $d r f d u=\operatorname{sen}(K, O-P)$ per il vetrore unitz parallelo a $P_{v}^{\prime}$ si ha che $P_{v i}^{\prime \prime} P_{u, \psi}^{\prime \prime}=r \operatorname{sen}(K, O-P)$; si ha dunque

$$
\frac{d}{d s}\left(\frac{d P}{d s} \mid P_{v}^{\prime}\right)=\left.\frac{d^{2} P}{d s^{2}}\right|_{v} ^{\prime}
$$

che dimostra il teorema di Cla iraut,

$\mathrm{Si}$ osservi che si puo porre

$$
P=Q+r\left(I+e^{l v}\right) I,
$$

ove $Q$ descrive la semi meridiana, $r$ è la distanza di $Q$ dall'asse, $I$ é un vettore unita del piano della curva $Q$ normale all'asse, e l'operazione $i$ e fatta sur piani dei paralleli. 
Sia $Q$ un punto e $I$ un vettore unitd funzioni continue... di $u$ e v. La congruenza è descritta dalla retta $Q I$. Se $O$ e un punto fisso, $0+I$ descrive la rappresentaitione sferica della congruenza, e l'elemento di arco descritto da $O+I$ e

$$
d s=\bmod d I \text {. }
$$

Per una direzione $d I$, cioè per un valore di $d u \int d v$, si ha una superficie rigata descritta da $Q I$ : il piano centrale per la generatrice $Q I$ d

$$
Q I I(d I)=Q \mid d I
$$

e il punto centrale $R$ in $Q I$ è la posizione della forma

$$
\begin{gathered}
{[Q(d I)+(d Q) I][Q \mid d I]=-[(d I) Q \mid d I Q+[(d Q) Q \mid d I I=} \\
\frac{1}{6}[(d I \mid d I) Q-(d Q ! d I) I]
\end{gathered}
$$

e quindi

$$
R=Q-\frac{d Q \mid d I}{d l \mid d I} I=Q-\left(\frac{d Q \mid d I}{d s \mid d s}\right) I
$$

e se indichiano con $r$ la distanza di $R$ da $Q$ si ha

$$
r=-\frac{d Q \mid d I}{d ! d I}=-\frac{d Q \mid d I}{d s} \mid \frac{1}{d s}
$$

Sieno state scelte le variabili $u, v$ in guisa che $\mathrm{i}$ coefficienti medii delle due forme $d I \mid d I, d Q d d I$ sieno nulli, in guisa ciod che si abbia

$$
I_{*}^{\prime} I_{v}^{\prime}=0, \quad Q_{*}^{\prime} I_{v}^{\prime}+Q_{v}^{\prime} I_{*}^{\prime}=0
$$

le quali relazioni sussistono per qualunque punto $Q+x I$ della retta 
$Q I$, come è facile verificare. [Si ha, ad es.,

$$
(Q+x)_{s}^{\prime} \mid I_{n}^{\prime}=\left(Q_{u}^{\prime}+x I_{s}^{\prime}+x_{n}^{\prime} D\right) I_{v}^{\prime}=Q_{n}^{\prime}\left[I_{v}\right]
$$

Supposte verificate le ( 16$)$, poniamo

$$
r_{3}=-\frac{Q_{p}^{\prime} \mid I_{*}^{\prime}}{Y_{*}^{\prime} I_{*}^{\prime}}, \quad r_{3}=-\frac{Q_{0}^{\prime} I_{0}^{\prime}}{I_{*}^{\prime} \mid I_{v}^{\prime}}
$$

e $Q+r_{1} I, Q+r_{2} I$ sono i punti centrali in $Q I$ delle due rigate corrispondenti a $v=$ cost. ed $u=$ cost.

Dalla (15), (16) e (17) si ha

$$
r=-Q_{a}^{\prime} I_{u}^{\prime}\left(\frac{d u}{d s}\right)^{2}-Q_{v}^{\prime} I_{v}^{\prime}\left(\frac{d v}{d s}\right)^{2}=r_{1}\left(I_{u}^{\prime}\right)^{2}\left(\frac{d u}{d s}\right)^{2}+r_{2}\left(I_{v}^{\prime}\right)^{2}\left(\frac{d v}{d s}\right)^{2}
$$

e detto $\varphi$ l'angolo che $d I$ fa con $I$ si ha $\left(n^{\circ} 3\right)$

$$
r=r_{1} \cos ^{2} \varphi+r_{2} \operatorname{sen}^{2} \varphi
$$

che è la formula di Hamilton; dalla quale deducesi che $r_{1}, r_{2}$ sono i massimi e minimi valori di $r$. Ma $d \Pi d I$ e unz forma definita e quindi dalla ( 5 ) risulta che $r$ acquista effettivamente un valor massino $e$ un valor minimo : in conseguenza si possono sempre scegliere le $n, v$ (in un sol modo, $O$ infiniti modi secondoche $r_{1} \neq r_{2}$ o $r_{1}=r_{2}$ ) in guisa che sieno verificate le (16) e $r_{1}, r_{1}$ sono indipendenti dal sistema di coordinate $u, v$. Le sup. rigate descritte da $Q I$ e corrispondenti ad $u=$ cost. e $v=$ cost., che diconsi principali, hanno i piani centrali per $Q I$ ortogonali e il piano centrale dell'una e asintotico per l'altra.

Se nella (i 8 ) poniamo al posto di $\varphi, \frac{\pi}{2}-\varphi \circ \varphi+\frac{\pi}{2}$ i valori di $r$ sono eguali e detto $r^{\prime}$ uno di questi si ha

$$
r^{\prime}=r_{3} \operatorname{sen}^{2} \varphi+r_{2} \cos ^{2} \varphi
$$

Rend. Circ. Matem., t. XII, parte $1^{*}$.-Stampato il 29 marzo 1898. 
che combinata con la (t8) da

$$
r+r^{\prime}=r_{2}+r_{2}
$$

la quale prova che $i$ punti centrali corrispondenti a due direzioni ortogonali, o a due direzioni aventi con $i$ piani centrali principali $i$ bisctori a comune, sono equidistanti dal punto

$$
P=Q+\frac{r_{1}+r_{2}}{2} I
$$

che dicesi centro della retta $Q I$ nella congruenza.

Considerando la congruenza generata dalla retta $P I$, le formule (16) sussistono ancora ove al posto di $Q$ si legga $P$ : posto (supponendo $r_{2} \supseteq r_{1}$ )

$$
2 b=r_{1}-r_{1},
$$

le (17) danno

$$
\frac{P_{u}^{\prime} \mid I_{*}}{I_{*}^{\prime} \mid I_{*}}=-\frac{P_{n}^{\prime} \mid I_{v}}{I_{v}^{\prime} \mid I_{*}}=b
$$

e la formula di Hamilton diviene

$$
r=b \cos 2 \varphi ;
$$

dalla quale, come del resto anche dalla ( 18 ), deducesi che corrispondentemente alle direzioni $d I$ che bisecano $I_{\text {, e }}^{\prime} I_{\text {, si hanno due }}$ rigate che hanno $P$ per punto centrale.

Per $b=0$ si hanno le congruenze isotrope di $R i b a u c o u r$, per le quali ogni rigata es principale e per ognuna di esse il punto centrale sulla retta $P I$ e il punto $P$.

Per un punto qualunque $S$ della retta $P I$ si ha

$$
S=P+x I
$$


SOPRA ALCUNE QUUSTIONI di GBONETRIA DIPPERENZIALE. IjI ove $x$ é un numero funzione di $u, v$. Se $x$ è tale che il vettore

$$
d S=d P+x d I+(d x) I
$$

e normale, qualunque sia $d u / d v$, ad $I$, allora la congruenza d normale. Ora $d S \mid I=0$ quando

$$
d x=-d P \mid I,
$$

perchè essendo $I$ un vettore unith si ba che $I \mid d I=0$. La precedente condizione assume la forma

$$
d x=-\left(P_{s}^{\prime} \mid I\right) d u-\left(P^{\prime} \mid I\right) d v,
$$

la quale e sodisfatta quando

$$
\frac{d}{d v}\left(P_{*}^{\prime} \mid I\right)=\frac{d}{d u}\left(P_{*}^{\prime} \mid I\right),
$$

cioè quando

$$
P^{\prime}\left|I_{v}^{\prime}=P_{*}^{\prime}\right| I_{*}^{\prime}
$$

che combinata con la seconda delle (16) da

$$
P_{\|}^{\prime}\left|I_{*}^{\prime}=P_{v}^{\prime}\right| I_{*}^{\prime}=0
$$

Queste, essendo ortogonali i vettori $I_{w}^{\prime}, I_{v}^{\prime}$ e perpendicolari ad $I$, dice che i vettori $I, P_{4}^{\prime}, I_{4}^{\prime}$ sono complanari e sono pure complanari i vettori $I, P_{v}^{\prime}, I_{v}^{\prime}:$ ma poichè l'equazione differenziale delle rigate sviluppabili della congruenza e $d(P I) \cdot d(P I)=0$, ovvero I.dP.dI=0, si ha che: una congruenza \& normale solo quando le sue due sup. principali sono sviluppabili.

Se indichiamo con $\propto$ e $\beta$ gli angoli che $l$ fà, rispettivamente,

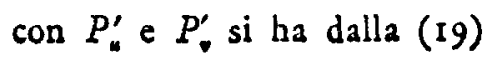

$$
d x=-\left(\bmod P_{v}^{\prime}\right) \cos \alpha d u-\left(\bmod P_{v}^{\prime}\right) \cos \beta d v,
$$


132

C. BURAII-PORTt.

dalla quale si deduce subito il teorema di Beltrami, che esprime rimanere la sup. $S$ ortogonale ai raggi della congruenza per qualunque flessione della sup. $P$. Dalla (20) espressa mediante $\alpha$ e $\beta$ si deduce, con $i$ metodi ordinari, il teorema di Malus-Dupin che esprime rimanere normale ogni congruenza normale dopo aver subito un numero qualunque di rillessioni o di rifrazioni.

Torino, gennajo 1898 .

C. BURALI-FORTI. 